PROCEEDINGS OF THE

AMERICAN MATHEMATICAL SOCIETY

Volume 128, Number 2, Pages 453-462

S 0002-9939(99)04987-4

Article electronically published on September 27, 1999

\title{
NONSYMMETRIC SYSTEMS AND AREA INTEGRAL ESTIMATES
}

\author{
G. C. VERCHOTA AND A. L. VOGEL
}

(Communicated by Christopher D. Sogge)

\begin{abstract}
Even though the $L^{2}$ Dirichlet problem on Lipschitz domains is not always solvable for nonsymmetric strongly elliptic systems, so that many results and techniques from the symmetric systems are unavailable, there are some similarities with the symmetric systems. We show that the nontangential maximal function and the square function of a solution are equivalent and that there is a Fatou theorem for these solutions.
\end{abstract}

\section{INTRODUCTION}

The systems we will be working with are second order, nonsymmetric, strongly elliptic systems with real constant coefficients which consist of two equations in two unknowns in the plane (3). For the model skewsymmetric systems (4), the system may be written in the complex form (6) $\partial \bar{\partial}_{k} u=0$. The parameter $k$ is the canonical ellipticity, $0<k \leq 1$ with $k=1$ giving an uncoupled system of Laplacians. The solutions have the form $(7) u(z)=f((1+k) z-(1-k) \bar{z})+\overline{g(z)}=\left(f_{k}+\bar{g}\right)(z)$ where $f$ and $g$ are holomorphic. In [VV97, Theorem 6.11] the authors showed that the Dirichlet problem for the nonsymmetric systems is solvable on $C^{1}$ polygons for $p$ large depending on the corners in the domain. They also obtained the corresponding results for the Regularity [VV97, Theorem 6.9] and Neumann problems [VV97, Theorem 6.5]. In addition, they showed that the weak maximum principle holds in $C^{1}$ polygons [VV97, Theorem 7.3]. The material used to obtain these positive results are explicit formulas for the fundamental solution (8) and its conormal derivative, the construction of a conjugate solution (14) satisfying the CauchyRiemann equations Theorem 2.1, and a Poisson representation for solutions (16) on smooth domains.

The most striking result of nonsymmetry is that the $L^{2}$ Dirichlet problem which is always solvable for symmetric systems on Lipschitz domains is not always solvable for nonsymmetric systems on domains with just a single corner. Indeed, the lack of solvability in $L^{p}$ may occur for any $p<\infty$. The values of $p$ for which solvability fails, which are between 1 and 2 for the symmetric systems, move to 2 and above. It should be noted that when $0<k<1$, even for symmetric systems, these values of $p$ depend on the orientation of the corner (see [VV97, (42) or (65)]). All this

Received by the editors September 16, 1997 and, in revised form, March 16, 1998.

1991 Mathematics Subject Classification. Primary 35J55, 31A25.

Key words and phrases. Elliptic, bianalytic, area integral.

The first author was partially supported by NSF Grant DMS-9706648.

(C)1999 American Mathematical Society 
is demonstrated by explicit formulas for solutions in corners which go back to [Koz90]. For directions $\alpha, \beta \in \mathbb{C}$ with $\arg \alpha<\arg \beta$ a corner domain is given by $\{\zeta \in \mathbb{C} \mid \arg \alpha<\arg \zeta<\arg \beta\}$ and the solutions which lead to counterexamples to uniqueness in the skewsymmetric case have the form

$$
u(\zeta)=e^{-2 \pi i z}\left(\frac{\bar{\zeta}}{\bar{\alpha}}\right)^{z}-\left(\frac{(1+k) \zeta-(1-k) \bar{\zeta}}{(1+k) \alpha-(1-k) \bar{\alpha}}\right)^{z}
$$

for a suitably chosen parameter $z=-\frac{1}{p}+i \xi$ (see [VV97, §8] and [CD93]). The lack of solvability on Lipschitz domains may be thought of as the result of the lack of boundary energy estimates known as Rellich identities which appear to be impossible to obtain, simply by integration by parts, for the skewsymmetric systems even in the case of a flat boundary. However, for the skewsymmetric systems there is a range of ellipticity $k_{0}<k<1$ for which there are, as yet, no counterexamples in Lipschitz domains nor solvability results in Lipschitz domains. But even for this restricted set of skewsymmetric systems there does not appear to be a way to "get to the boundary" to prove solvability in $L^{p}$ on Lipschitz domains. That is, we are unable to bound the nontangential maximal function of a solution by its boundary values in $L^{p}$. A natural question then is, does a solution with nontangential maximal function in $L^{p}$ have nontangential limits almost everywhere, i.e. is there a Fatou type theorem for these solutions? Since we have available a Green's formula representation (11), assuming smoothness, of a solution as a sum of a double layer potential of the solution and a single layer potential of the conormal derivative of the solution the answer would be yes if the square function of the solution is dominated by the nontangential maximal function of the solution.

Recently $\left[\mathrm{DK}^{+}\right]$have obtained the $L^{p}$ equivalence of the nontangential maximal function and the square function for solutions to the homogeneous problem for higher order, symmetric, elliptic systems with real constant coefficients on Lipschitz domains in $\mathbb{R}^{n}$. Closely related to this is [KKPT98], which contains results for nonsymmetric equations. These authors show that the equivalence between area integrals and nontangential maximal functions implies (in any dimension) an $A^{\infty}$ estimate on harmonic measure. Our interest is in obtaining the equivalence for nonsymmetric strongly elliptic systems. But $\left[\mathrm{DK}^{+}\right]$relies on the fact that one can get to the boundary (via Rellich formulas [PV95]). Unable to do this we show directly in $\S 3$, that the nontangential maximal functions of a solution and an appropriately chosen conjugate solution are equivalent in $L^{p}$ on Lipschitz domains. For this we use ideas of Dahlberg, Kenig and David [Ken86, Lemma 3.1.11]. The Fatou theorem follows (without using the square function), the result of functional analysis arguments using the Green's formula, properties of conjugate solutions viz.

$$
u=\mathcal{K} u+\left(\mathcal{S} \frac{\partial}{\partial \tau}\right) \widetilde{u},
$$

and the equivalence of the nontangential maximal functions.

Theorem 1.1 (Fatou). Let $u$ be a solution to a nonsymmetric strongly elliptic system (3) on a bounded Lipschitz domain $\Omega$. If $\mathcal{N}(u) \in L^{p}(\partial \Omega)$ for $1<p<\infty$, then $u$ has a nontangential limit $g \in L^{p}(\partial \Omega)$ almost everywhere on $\partial \Omega$.

In $\S 4$ we show that the nontangential maximal function and the square function for a solution are equivalent in $L^{p}$ on bounded Lipschitz domains given the 
normalization that the solution is zero at some point in the domain. That the nontangential maximal function is dominated by the square function follows from (1) and the argument in $\left[\mathrm{DK}^{+}\right]$. For the other direction, the argument in $\left[\mathrm{DK}^{+}\right]$relies on Rellich formulas which are unavailable to us. Instead we use the representation of the solutions involving holomorphic functions (7), harmonic function results, and the equivalence of the nontangential maximal functions from $\S 3$.

\section{Preliminaries}

Let $A, B$, and $C$ be real, constant $2 \times 2$ matrices. The general system we are concerned with has the form

$$
\left(A \frac{\partial^{2}}{\partial x^{2}}+2 B \frac{\partial^{2}}{\partial x \partial y}+C \frac{\partial^{2}}{\partial y^{2}}\right)\left(\begin{array}{l}
u(x, y) \\
v(x, y)
\end{array}\right)=\left(\begin{array}{l}
0 \\
0
\end{array}\right)
$$

for real functions $u$ and $v$. The strongly elliptic, nonreducible systems may be transformed into

$$
\left(\left(\begin{array}{cc}
1 & 0 \\
0 & \frac{\lambda}{k^{2}}
\end{array}\right) \frac{\partial^{2}}{\partial x^{2}}+\left(\begin{array}{cc}
0 & \frac{\lambda-k^{2}}{k} \\
\frac{\lambda-1}{k} & 0
\end{array}\right) \frac{\partial^{2}}{\partial x \partial y}+\left(\begin{array}{cc}
\lambda & 0 \\
0 & 1
\end{array}\right) \frac{\partial^{2}}{\partial y^{2}}\right)\left(\begin{array}{l}
u \\
v
\end{array}\right)=\left(\begin{array}{l}
0 \\
0
\end{array}\right)
$$

where $\lambda>0, \lambda \neq 1, k^{2}$ and $0<k \leq 1$ [KWCQ85, Chapter 1, Section 4]. The parameter $k$ is a measure of the ellipticity, and $\lambda$ a measure of symmetry. The nonsymmetric strongly elliptic systems are obtained by choosing $\lambda$ between $k^{2}$ and 1 ; the other values of $\lambda>0$ give systems which may be transformed into symmetric strongly elliptic systems. For clarity and ease of presentation we will work with the systems obtained from (3) by choosing $\lambda=k$ and multiplying the second equation by $k$ :

$$
\left(\left(\begin{array}{ll}
1 & 0 \\
0 & 1
\end{array}\right) \frac{\partial^{2}}{\partial x^{2}}+\left(\begin{array}{cc}
0 & 1-k \\
k-1 & 0
\end{array}\right) \frac{\partial^{2}}{\partial x \partial y}+\left(\begin{array}{ll}
k & 0 \\
0 & k
\end{array}\right) \frac{\partial^{2}}{\partial y^{2}}\right)\left(\begin{array}{l}
u \\
v
\end{array}\right)=\left(\begin{array}{l}
0 \\
0
\end{array}\right)
$$

We call these the skewsymmetric systems. The other nonsymmetric systems, $k<$ $\lambda<1$, present only technical difficulties (see [VV97]). Those with $k^{2}<\lambda<$ $k$ may be transformed into systems with $k<\lambda<1$. Strong ellipticity for the skewsymmetric systems (4) is then simply that the Legendre-Hadamard condition

$$
a_{i j}^{\alpha \beta} \eta^{\alpha} \xi_{i} \eta^{\beta} \xi_{j}=\left(\xi_{1}^{2}+k \xi_{2}^{2}\right)\left(\eta_{1}^{2}+\eta_{2}^{2}\right) \geq k|\xi|^{2}|\eta|^{2}
$$

holds for all nonzero $\xi=\left(\xi_{1}, \xi_{2}\right)$ and $\eta=\left(\eta_{1}, \eta_{2}\right)$ in $\mathbb{R}^{2}$ where $a^{11}=\left(\begin{array}{ll}1 & 0 \\ 0 & k\end{array}\right)=a^{22}$, $a^{12}=\left(\begin{array}{cc}0 & 1-k \\ 0 & 0\end{array}\right)$ and $a^{21}=\left(\begin{array}{cc}0 & 0 \\ k-1 & 0\end{array}\right)$.

In $\S 3$ it will be convenient to work with the system in yet a different form in which many of the algebraic manipulations of matrices are reduced to multiplication of complex numbers. Setting $w=u+i v$, and using the complex derivatives $\partial=$ $\frac{1}{2}\left(\frac{\partial}{\partial x}-i \frac{\partial}{\partial y}\right)$ and $\bar{\partial}=\frac{1}{2}\left(\frac{\partial}{\partial x}+i \frac{\partial}{\partial y}\right)$ we rewrite (4) as the single complex equation

$$
L w=2 \partial \bar{\partial}_{k} w=0
$$

where $\bar{\partial}_{k}=(1+k) \bar{\partial}+(1-k) \partial$. The regular solutions of (6) have an especially nice form. For any holomorphic functions $f$ and $g$ set $f_{k}(z)=f((1+k) z-(1-k) \bar{z})$; then $\bar{\partial}_{k} f_{k}(z)=0$ and $\partial \overline{g(z)}=0$. The form is

$$
f_{k}(z)+\overline{g(z)}
$$

(see the comments following equation (50) in [VV97] or [KWCQ85, Chapters 2 and $3])$. 
The fundamental solution operator for $(6)$ is

$$
\Gamma(\zeta)=\frac{1}{2(1+k) \pi}\left(\log |\zeta|^{2}+\log \left(\frac{1+k}{2}-\frac{1-k}{2} \frac{\bar{\zeta}}{\zeta}\right)\right)
$$

using the principal branch of the logarithm.

Let $\Omega$ be any bounded Lipschitz domain in the plane, i.e. for any point $P \in \partial \Omega$ there is a neighborhood $U$ of $P$, a Lipschitz function $\phi: \mathbb{R} \rightarrow \mathbb{R}$ and a rectangular coordinate system $(s, t)$ so that $U \cap \Omega=U \cap\{(s, t) \mid t>\phi(s)\}$. Here $\phi$ being Lipschitz means $\left|\phi\left(s_{1}\right)-\phi\left(s_{2}\right)\right| \leq b\left|s_{1}-s_{2}\right|$ for all $s_{1}, s_{2}$ and some $b<\infty$. The smallest such $b$ is the Lipschitz norm of $\phi$ and is denoted by $\|\nabla \phi\|_{\infty}$. The outer unit normal vector $N_{P}$ is defined a.e. with respect to surface measure $d \sigma(P)$ on $\partial \Omega$. We write these normals in complex notation as $N=N_{1}+i N_{2}$. For each operator $L=2 \partial \bar{\partial}_{k}$ we define the conormal derivative at the boundary by

$$
\frac{\partial}{\partial \nu}=\bar{N} \bar{\partial}_{k}-i \frac{\partial}{\partial \tau}
$$

where the tangential derivative $\partial / \partial \tau$ is defined by

$$
\frac{\partial}{\partial \tau}=-i \frac{1+k}{2}(\overline{N \partial}-N \partial)
$$

Let $\Omega$ be a bounded domain with a sufficiently smooth boundary, $\partial \Omega$, and let $v$ be a sufficiently smooth function; then we have a Green's formula

$$
v(\xi)=\int_{\partial \Omega} \frac{\partial \Gamma}{\partial \nu}(\zeta-\xi) v(\zeta)-\Gamma(\zeta-\xi) \frac{\partial v}{\partial \nu}(\zeta) d \sigma(\zeta)+\int_{\Omega} \Gamma(\zeta-\xi) L v(\zeta) d x d y
$$

where $\zeta=x+i y$. For any $1<p<\infty$ and $f \in L^{p}(\partial \Omega)$ we have the double layer potential

$$
\mathcal{K} f(\xi)=\int_{\partial \Omega} \frac{\partial \Gamma}{\partial \nu}(\zeta-\xi) f(\zeta) d \sigma(\zeta), \quad \xi \in \mathbb{C} \backslash \partial \Omega
$$

and the single layer potential

$$
\mathcal{S} f(\xi)=\int_{\partial \Omega} \Gamma(\zeta-\xi) f(\zeta) d \sigma(\zeta), \quad \xi \in \mathbb{C}
$$

Next we define the conjugate solutions and a conjugate operator to $\mathcal{K}$. Let $f$ and $g$ be holomorphic and recall the regular solutions (7) have the form $u=f_{k}+\bar{g}$. Now $f$ and $g$ are uniquely determined if we require that $g\left(\xi_{0}\right)=0$ for some fixed point $\xi_{0}$ in the domain. With this normalization, the conjugate solution $\widetilde{u}$ is

$$
\widetilde{u}(\xi)=-i f_{k}(\xi)+i \overline{g(\xi)} .
$$

Note that $\widetilde{\widetilde{u}}=-u$. We also have the rotation invariant Cauchy-Riemann system

Theorem 2.1 ([VV97]). Given a solution $u$ and any direction $N \in \mathbb{C}$ with conormal and tangential derivatives defined by (9) and (10) we have

$$
\frac{\partial}{\partial \nu} u=\frac{\partial}{\partial \tau} \tilde{u} \quad \text { and } \quad \frac{\partial}{\partial \tau} u=-\frac{\partial}{\partial \nu} \tilde{u}
$$

In addition, for $N$ fixed, the conjugate of $\frac{\partial}{\partial \tau} u$ is $\frac{\partial}{\partial \nu} u$, i.e., both are solutions and

$$
\frac{\partial}{\partial \nu} u=\widetilde{\frac{\partial}{\partial \tau}} u .
$$


Given a solution $u$ Theorem 2.1 provides the standard local algorithm for arriving at $\tilde{u}$, viz. integrating $\frac{\partial}{\partial \nu} u$ in the $\tau$ direction, a fact we shall rely on in $\S 3$. Another method that is global in nature is to define the potential

$$
\left(\mathcal{S} \frac{\partial}{\partial \tau}\right) f(\xi)=\int_{\partial \Omega} \frac{\partial \Gamma}{\partial \tau_{\zeta}}(\zeta-\xi) f(\zeta) d \sigma(\zeta) .
$$

Then the conjugate of $\left(\mathcal{S} \frac{\partial}{\partial \tau}\right) f$ is the double layer potential $\mathcal{K} f$.

Finally, we have a Poisson representation for solutions on smooth domains, from [VV97, (51) and (59)]

$$
u(\xi)=4 \int_{\partial \Omega} \frac{\partial}{\partial \nu_{\zeta}} G^{\xi}(\zeta) u(\zeta) d \sigma(\zeta)
$$

\section{Nontangential maximal FUnCtions ARE EQUivalent in $L^{p}$}

To begin, we need some notation and terminology. Let $\Omega$ be a bounded Lipschitz domain and $P \in \partial \Omega$. The nontangential approach region at $P$ is

$$
\Gamma(P)=\{z \in \Omega|| z-P \mid \leq(1+\alpha) \operatorname{dist}(z, \partial \Omega)\}
$$

where $\alpha>0$ will be chosen large enough depending on the Lipschitz nature of the boundary, which is defined below. The nontangential maximal function of a function $u$ on $\Omega$ is defined for $P \in \partial \Omega$ by

$$
\mathcal{N}(u)(P)=\sup _{\Gamma(P)}|u|
$$

The nontangential limit of $u$ at $P$ is the limit of $u$ as $z$ approaches $P$ through points in $\Gamma(P)$ and is denoted by $u(z) \stackrel{\text { n.t. }}{\rightarrow} u(P)$. It is a well known consequence of [CMM82] and [Cal77] that $\mathcal{K} f \stackrel{\text { n.t. }}{\rightarrow}\left(\frac{1}{2} I+K\right) f$ where $K$ is the principal value operator obtained from (12) by allowing $\xi \in \partial \Omega$, and further that $\|\mathcal{N}(\mathcal{K} f)\|_{p} \leq$ $C_{p}\|f\|_{p}$ when $1<p<\infty$. Similarly the trace of (15) is its principal value with $\left\|\mathcal{N}\left(\mathcal{S} \frac{\partial}{\partial \tau}\right) f\right\|_{p} \leq C_{p}\|f\|_{p}$. See $\S \S 3$ and 5 of [VV97].

We will say that two positive quantities $R$ and $S$ are equivalent if there are constants $C_{1}$ and $C_{2}$ so that $R \leq C_{1} S$ and $S \leq C_{2} R$. The constants $C_{1}, C_{2}$ will be called constants of comparability. The constants of comparability between the $L^{p}$ norms of the nontangential maximal functions will depend on the nontangential approach regions through the parameters $\alpha$ and $\widehat{\alpha}$, chosen below, and a Lipschitz nature for $\partial \Omega$ to which we now turn our attention. Given a bounded Lipschitz domain and a point $P \in \partial \Omega$ there is a rectangular coordinate system $(s, t)$ centered at $P$ and a cylinder $Z$ centered at $P$ so that

(i) the axis of the cylinder $Z$ is along the $t$-axis,

(ii) the intersection of the cylinder with the $s$-axis is the interval $[-r, r]$,

(iii) there is a Lipschitz function $\phi(s)$ with $Z \cap \partial \Omega=\{(s, \phi(s)) \mid-r \leq s \leq r\}$, and $Z \cap \Omega=\{(s, t) \in Z \mid t>\phi(s)\}$,

(iv) the top of the cylinder is at $10\left(\|\nabla \phi\|_{\infty}+1\right) r$ on the $t$-axis.

This cylinder is denoted by $Z(P, r)$. In general $Z(P, \eta r)=\eta Z(P, r)$ is the dilation of $Z(P, r)$ about $P$ by $\eta$. By compactness a finite number $N$ of the $Z(P, r / 4)$ cover $\partial \Omega$ and we may use the same $r$ for every $P$. We let $M$ be the maximum of the Lipschitz norms of the associated functions $\phi$. We may now replace (iv) by

(iv) the top of the cylinder, for any $P$, is at $10(M+1) r$ on the $t$-axis 
at the expense of making $r$ possibly smaller and $N$ possibly larger. A Lipschitz nature for $\partial \Omega$ then includes both $M$ and $N$. $M$ will be called a Lipschitz norm for $\Omega$ or $\partial \Omega$. We also note that $Z(P, r) \cap \Omega$ is starlike with respect to any point $(s, t) \in Z(P, r)$ with $t>3 M r$.

Remark 1 . We make a few more observations, by choosing $\alpha$ large enough in the definition of the nontangential approach regions all the points $(s, t) \in Z(P, r / 4)$ with $t>r(M+1)$ will be contained in $\Gamma(P)$ for all $P \in \partial \Omega \cap Z(P, r / 4)$, and by choosing $\alpha$ possibly larger still $\Gamma(P)$ is a connected set for all $P \in \partial \Omega$. Finally, set $A_{P}$ to be the center of the top of $Z(P, r / 4)$.

Let $u$ and $\widetilde{u}$ be solutions that satisfy the Cauchy-Riemann system on $\Omega$ (see Theorem 2.1). In order to show that their nontangential maximal functions are equivalent a normalization is required, for some $A \in \Omega$ we require that $u(A)=$ $\widetilde{u}(A)=0$.

Remark 2. The constants of comparability will depend on the point $A$ through the parameter $\widehat{\alpha}$ used to define the nontangential approach regions $\widehat{\Gamma}$ below. We make a preliminary choice of $\widehat{\alpha}>2 \alpha$ and so that $A \in \widehat{\Gamma}(P)$ for every point $P \in \partial \Omega$.

Remark 3. As a consequence, there is a polygonal path from $A_{P}$ to $A$ contained in $\widehat{\Gamma}(P)$ that is covered by a finite number $N^{\prime}$ of balls contained in $\widehat{\Gamma}(P)$ of radius $c_{1} r$. The constants $N^{\prime}$ and $c_{1}$ depend only on $M, N$, and $\widehat{\alpha}$.

Theorem 3.1. Let $L u=0$ on the bounded Lipschitz domain $\Omega, 0<p<\infty$, and let $\widetilde{u}$ be a conjugate solution with $u(A)=\widetilde{u}(A)=0$ for some $A$ in $\Omega$. Then there is a finite constant $C$ that depends on $M, N, p, A, \alpha$ and the ellipticity of $L$ so that

$$
\|\mathcal{N}(\widetilde{u})\|_{p} \leq C\|\mathcal{N}(u)\|_{p} .
$$

Of course, this gives the equivalency as we may interchange $u$ with $\tilde{u}$.

Proof. The argument is patterned after one in [Ken86, Lemma 3.1.11] due to Dahlberg, Kenig, and David. First (19) is proved in the case of domains with a small Lipschitz norm and then a build-up scheme is followed to obtain (19) for all Lipschitz domains. Since we have nonsymmetric systems with a solution and its conjugate to compare instead of a symmetric system with antisymmetric and symmetric parts of a gradient to compare, we check to see that the relevant part, (23), of the argument goes through.

We work with $p=2$, the other values of $p>0$ being handled by an argument that goes back to [BG72]. For domains with small Lipschitz norm the trace of the double layer potentials $\pm \frac{1}{2} I+K$ are known to be Fredholm because $K$ may be seen as a small norm (see [Cal77] and [FJR78]) plus compact. Consequently all boundary value problems of $\S 5$ [VV97] regarding existence and uniqueness of solutions follow in the small Lipschitz case. Therefore, if $\mathcal{N}(u) \in L^{2}$ we have that $u$ restricted to parallel boundaries (see [Ver84, Thm 1.12]) is uniformly in $L^{2}$ so that an elementary weak convergence argument with the Poisson kernel (16) establishes the existence of $L^{2}$ boundary values for $u$. Then using (1) similarly on parallel boundaries with the roles of $u$ and $\widetilde{u}$ reversed and using the invertibility of $\frac{1}{2} I-K$ and the boundedness of (15) the $\widetilde{u}$ are uniformly bounded on parallel boundaries by $\|\mathcal{N}(u)\|_{2}$. Then (1) may be established on $\partial \Omega$ and $\|\mathcal{N}(\widetilde{u})\|_{2} \leq C\|\mathcal{N}(u)\|_{2}$ follows from the nontangential maximal properties of $\mathcal{K}$ and $\mathcal{S} \frac{\partial}{\partial \tau}$. 
Now we build up the Lipschitz norms. Suppose that (19) holds for domains with Lipschitz norm $\leq M$ and let $\Omega$ be a bounded Lipschitz domain with norm $\leq \frac{39}{38} M$. Suppose that $u$ is a solution with $\mathcal{N}(u) \in L^{2}(\partial \Omega)$. For $t>0$ set $E_{t}=$ $\{\mathcal{N}(\widetilde{u})>t\}$ and let $\left\{\Delta_{j}\right\}$ be a Whitney decomposition of the open set $E_{t}$ into closed, nonoverlapping, connected boundary pieces so that

(i) each $\Delta_{j}=Z\left(P_{j}, r / 2^{k_{j}}\right) \cap \partial \Omega$ for some positive integer $k_{j} \geq 2$.

(ii) if $\left|\Delta_{j}\right|<c_{2} r$, there is a point $z_{j} \in \partial \Omega \cap Z\left(P_{j}, r / 2^{k_{j}-1}\right)$ with $\mathcal{N}(\widetilde{u})\left(z_{j}\right) \leq t$. Moreover, $\operatorname{dist}\left(z_{j}, \Delta_{j}\right)$ is equivalent to $\left|\Delta_{j}\right|$. The constant $c_{2}$ depends only on $M$.

Note that to prove (19) we may use nontangential approach regions $\widehat{\Gamma}$ (see remark 2 ), that have a wider opening on the right-hand side of (19) (see [FS72]). Denote the associated nontangential maximal function by $\widehat{\mathcal{N}}$. Given $\beta>1$ and $\delta>0$ set $E_{j}=\Delta_{j} \cap\{\mathcal{N}(\widetilde{u})>\beta t, \widehat{\mathcal{N}}(u) \leq \delta t\}$. We claim that $\delta$ may be chosen so small that

$$
\left|E_{j}\right| \leq c(M)\left|\Delta_{j}\right| \text { for all } j
$$

where $c(M)<1$ depends only on $M$ and will be chosen below. With a $\delta$ in hand, we need only consider those $\Delta_{j}$ for which (20) is not true and show that by choosing $\delta$ smaller (20) holds. We assume that

$$
\left|E_{j}\right|>c(M)\left|\Delta_{j}\right| .
$$

We now form a sawtooth region $D_{j}$ above $E_{j}$ using the $\widehat{\Gamma}$ with $\widehat{\alpha}$ possibly larger, depending on $M$, so that $\partial D_{j}$ above $\Delta_{j}$ is the graph of a Lipschitz function $\widehat{\phi}$ with norm $\leq \frac{20}{19} M$. Let $s=\operatorname{diam}\left(\Delta_{j}\right)$; this sawtooth region satisfies

(i) $\bigcup_{P \in E_{j}}\left(\widehat{\Gamma}(P) \cap B\left(P, c_{3} s\right)\right) \subset D_{j} \subset \bigcup_{P \in E_{j}}\left(\bar{\Gamma}(P) \cap B\left(P, c_{4} s\right)\right)$ for constants $c_{3}$, $c_{4}$ independent of $j$.

(ii) $\partial D_{j} \cap \partial \Omega=E_{j}$.

(iii) $D_{j}$ is starlike and diameter of $D_{j}$ is equivalent to $s$.

Next we apply David's lemma [Jou83, page 113] to $\partial D_{j}$ above $\Delta_{j}$ to obtain the graph of a Lipschitz function $\psi$ (in a rotated coordinate system) with norm $\leq M$ such that the graph of $\psi$ is above the graph of $\widehat{\phi}$ (in the coordinate system of $\widehat{\phi}$ ) and so that the two graphs coincide on a set of significant measure independent of the Lipschitz norms; i.e. there is an absolute constant (independent of $M$ ) $0<\eta<1$ so that the measure of the coincidence set is at least $\eta$ of the measure of the graph of $\widehat{\phi}$. Let $F_{j}$ be that part of the coincidence set that is in $E_{j}$. It follows from an elementary consideration of the measures of sets that by choosing $c(M)$ close to 1 in (21) there is a constant $c_{5}>0$ so that

$$
\left|F_{j}\right| \geq c_{5}\left|E_{j}\right| .
$$

We now form a region $D_{j}^{\prime} \subset D_{j}$ so that

(i) $\partial D_{j}^{\prime}$ contains the graph of $\psi$ and so $F_{j}$.

(ii) $D_{j}^{\prime}$ has Lipschitz norm $\leq M$ and Lipschitz nature independent of $j$.

(iii) $D_{j}^{\prime}$ is starlike, $\operatorname{diam}\left(D_{j}^{\prime}\right)$ is equivalent to $s, D_{j}^{\prime}$ contains a starcenter $A^{\prime}$ whose distance to $\partial D_{j}^{\prime}$ is equivalent to $s$.

(iv) For all $P \in F_{j}, \Gamma(P) \cap B\left(P, c_{6} s\right) \subset D_{j}^{\prime} \backslash\left\{A^{\prime}\right\}$, where $c_{6}$ depends on $M, N, \alpha$ and is independent of $j$. 
On $D_{j}^{\prime}$ we use the nontangential approach regions $\Gamma^{\prime}$ with opening $\alpha^{\prime}$ depending on $M, N, \alpha$, so that for all $P \in F_{j}, \Gamma(P) \cap B\left(P, c_{6} s\right) \subset \Gamma^{\prime}(P) \cap B\left(P, c_{6} s\right)$. Let $\mathcal{N}^{\prime}$ be the nontangential operator for $D_{j}^{\prime}$; we claim that

$$
\mathcal{N}(\widetilde{u})(P) \leq \mathcal{N}^{\prime}(\widetilde{u})(P) \text { for all } P \in F_{j} .
$$

We now use the properties of our solutions and their conjugates. There are two cases depending on the size of $\left|\Delta_{j}\right|$. First, $\left|\Delta_{j}\right| \geq c_{2} r$ : Working from Remark 3, we integrate $\frac{\partial \widetilde{u}}{\partial \tau}$ along the path from $A$ to $A_{P}$ (see Remark 1). On each line segment $\tau$ is the direction of the line segment. The integrand is converted to $\frac{\partial u}{\partial \nu}$ using the Cauchy-Riemann equations. Now we use the Poisson representation of $u$ on balls contained in $\widehat{\Gamma}(P)$ and the fact that $|u| \leq \delta t$ in all of $\widehat{\Gamma}(P)$ to conclude, from the fundamental theorem of calculus and $\widetilde{u}(A)=0$, that $\left|\widetilde{u}\left(A_{P}\right)\right| \leq c \delta t$. The constant $c$ depends on $M, N, \alpha, A$ and on estimates of the gradient of the Poisson kernel in (16) when $|\xi-\zeta|$ is comparable to 1 . From $A_{P}$ there is similarly a path to any point in $\Gamma(P) \backslash B\left(P, c_{6} s\right)$. We conclude that $|\widetilde{u}| \leq c \delta t$ on all of $\Gamma(P) \backslash B\left(P, c_{6} s\right)$. (23) now follows in the first case by choosing $\delta<\beta / c$, and noting that $\mathcal{N}(\widetilde{u})>\beta t$ for all $P \in F_{j}$.

Second, $\left|\Delta_{j}\right|<c_{2} r$ : Then there is a nearby point $z_{j}$ (see (ii) in the description of $\left.\Delta_{j}\right)$, so that $\mathcal{N}(\widetilde{u})\left(z_{j}\right) \leq t$. This means $|\widetilde{u}| \leq t$ for all points in $\Gamma\left(z_{j}\right)$. For any $P \in F_{j}$, any point in $\left(\Gamma(P) \backslash B\left(P, c_{6} s\right)\right) \cap B\left(P, c_{7} r\right)$ may be joined by a polygonal path contained in $\widehat{\Gamma}(P)$ to a point in $\left(\Gamma\left(z_{j}\right) \backslash B\left(z_{j}, c_{6} s\right)\right) \cap B\left(z_{j}, c_{7} r\right)$. $c_{7}$ depends only on $M$. Each path may be covered by a finite number $N^{\prime \prime}$ of balls contained in $\widehat{\Gamma}(P)$ of the same radius. $N^{\prime \prime}$ depends only on $M, \alpha, \widehat{\alpha}$. Integrating along these paths as above, we conclude that $|\widetilde{u}| \leq t+c \delta t$ on $\left(\Gamma(P) \backslash B\left(P, c_{6} s\right)\right) \cap B\left(P, c_{7} r\right)$ for all $P \in F_{j}$. The remaining points in $\Gamma(P) \backslash B\left(P, c_{7} r\right)$ may now be reached from $\left(\Gamma(P) \backslash B\left(P, c_{6} s\right)\right) \cap B\left(P, c_{7} r\right)$ and the estimate $|\widetilde{u}| \leq t+c \delta t$ holds on all of $\Gamma(P) \backslash B\left(P, c_{6} s\right)$ for all $P \in F_{j}$. For $\delta$ small enough, depending on $\beta$ we see that $|\widetilde{u}|>\beta t$ is only possible on $\Gamma(P) \cap B\left(P, c_{6} s\right)$. (23) follows in the second case.

In the first case, we use (22) and the definition of $E_{j}$ to obtain

$$
c_{5}\left|E_{j}\right| \leq \int_{F_{j}}\left(\frac{\mathcal{N}(\widetilde{u})}{\beta t}\right)^{2} d \sigma .
$$

Using the claim we have

$$
c_{5}\left|E_{j}\right| \leq \int_{F_{j}}\left(\frac{\mathcal{N}^{\prime}(\widetilde{u})}{\beta t}\right)^{2} d \sigma \leq \int_{\partial D_{j}^{\prime}}\left(\frac{\mathcal{N}^{\prime}(\widetilde{u})}{\beta t}\right)^{2} d \sigma .
$$

At the expense of an additional term on the right-hand side of the form $c(\delta / \beta)^{2}\left|\Delta_{j}\right|$, due to adding and subtracting $\widetilde{u}\left(A^{\prime}\right)$ and the estimate $\left|\widetilde{u}\left(A^{\prime}\right)\right| \leq c \delta t$, we may assume that $u\left(A^{\prime}\right)=\widetilde{u}\left(A^{\prime}\right)=0$ at a star center $A^{\prime} \in D_{j}^{\prime}$. Since $D_{j}^{\prime}$ has Lipschitz nature, with norm $M$, independent of $j$ we may apply our theorem and use $|u| \leq \delta t$ on all of $D_{j}^{\prime}$ to obtain

$$
c_{5}\left|E_{j}\right| \leq c\left(\int_{\partial D_{j}^{\prime}}\left(\frac{\mathcal{N}^{\prime}(u)}{\beta t}\right)^{2} d \sigma+\left(\frac{\delta}{\beta}\right)^{2}\left|\Delta_{j}\right|\right) \leq c\left(\frac{\delta}{\beta}\right)^{2}\left|\Delta_{j}\right| .
$$

(20) follows in the first case by choosing $\delta$ small. 
In the second case, we note that $\mathcal{N}\left(\widetilde{u}-\widetilde{u}\left(A^{\prime}\right)\right) \geq \beta t-t-c \delta t>t(\beta-1) / 2$ for $\delta$ small enough and all $P \in F_{j}$. Then

$$
c_{5}\left|E_{j}\right|\left(t \frac{\beta-1}{2}\right)^{2} \leq \int_{F_{j}}\left(\mathcal{N}\left(\widetilde{u}-\widetilde{u}\left(A^{\prime}\right)\right)\right)^{2} d \sigma .
$$

Using (23) and applying our theorem on $D_{j}^{\prime}$

$$
c_{5}\left|E_{j}\right|\left(t \frac{\beta-1}{2}\right)^{2} \leq \int_{\partial D_{j}^{\prime}}\left(\mathcal{N}^{\prime}\left(u-u\left(A^{\prime}\right)\right)\right)^{2} d \sigma \leq c(\delta t)^{2}\left|\Delta_{j}\right| .
$$

The last inequality holds because $|u| \leq \delta t$ on all of $D_{j}^{\prime}$. Once again, (20) is obtained by choosing $\delta$ small.

From (20), standard distribution function arguments (see [Ken86, page 175]), and choosing $\beta$ the theorem follows.

\section{SquARE FUnCtions AND NONTANGENTIAL MAXimal FUnCTIONS}

The square function of a solution is defined by

$$
S(u)(P)=\left(\int_{\Gamma(P)}|\nabla u(\zeta)|^{2} d x d y\right)^{1 / 2}
$$

where $\zeta=x+i y$. We have the following theorem.

Theorem 4.1. Let $L u=0$ on a bounded Lipschitz domain $\Omega$ and $u(A)=0$ for some point $A \in \Omega$. Suppose that $\mathcal{N}(u) \in L^{p}(\partial \Omega)$ or $S(u) \in L^{p}(\partial \Omega)$ for $0<p<\infty$. Then there is a constant $C$ that depends only on the Lipschitz nature of $\Omega, p$ and A so that

$$
C^{-1} \int_{\partial \Omega}|\mathcal{N}(u)|^{p} d \sigma \leq \int_{\partial \Omega}|S(u)|^{p} d \sigma \leq C \int_{\partial \Omega}|\mathcal{N}(u)|^{p} d \sigma
$$

Proof. Again we work in $L^{2}$, the $L^{p}$ case being handled by the arguments in for example $\left[\mathrm{DK}^{+}, \S 4\right]$. For the inequality on the left, we first note that from (1) we may bound the $L^{2}$ norm of the nontangential maximal function of $u$ on smooth approximating domains by a constant that depends only on the Lipschitz nature of the domain times the sum of the $L^{2}$ norms of $u$ and $\widetilde{u}$. With the system as described following (5) we may use (5) and the argument in $\left[\mathrm{DK}^{+}, \S 2\right]$ to bound the $L^{2}$ norms of $u$ and $\widetilde{u}$ by their respective square functions. Finally, from the Cauchy-Riemann equations their square functions are the same, and the inequality follows. This inequality uses the assumption that $u(A)=0$.

For the inequality on the right we think of the solution as $u=f_{k}+\bar{g}$ where $f$ and $g$ are holomorphic. Then $|\nabla u|^{2} \leq 2\left(\left|\nabla f_{k}\right|^{2}+|\nabla g|^{2}\right)$ so that

$$
\begin{aligned}
\int_{\partial \Omega}|S(u)|^{2} d \sigma \leq c & \int_{\Omega}|\nabla u(\zeta)|^{2} \delta(\zeta) d x d y \\
& \leq c\left(\int_{\Omega}\left|\nabla f_{k}(\zeta)\right|^{2} \delta(\zeta) d x d y+\int_{\Omega}|\nabla g(\zeta)|^{2} \delta(\zeta) d x d y\right) .
\end{aligned}
$$

Here $\delta(\zeta)=\operatorname{dist}(\zeta, \partial \Omega)$. Recall that $f_{k}(\zeta)=f(T(\zeta))$ where $T(\zeta)=(1+k) \zeta-$ $(1-k) \bar{\zeta}$ is a linear transformation. With $\zeta=x+i y$ we have $T(\zeta)=2 k x+2 i y$. 
Also note that we may write $f_{k}$ and $\bar{g}$ using the solution and its conjugate,

$$
2 i f_{k}=i u-\widetilde{u} \text { and } 2 i \bar{g}=i u+\widetilde{u} .
$$

Using harmonic function results [Dah80] on the two integrals on the right of (29) and (30) we have

$$
\int_{\partial \Omega}|S(u)|^{2} d \sigma \leq c\left(\int_{\partial \Omega}|\mathcal{N}(u)|^{2} d \sigma+\int_{\partial \Omega}|\mathcal{N}(\widetilde{u})|^{2} d \sigma\right) .
$$

The result follows since these two nontangential maximal functions are equivalent.

\section{REFERENCES}

[BG72] D. Burkholder and R. Gundy, Distribution function inequalities for the area integral, Studia Math. 44 (1972), 527-544. MR 49:5309

[Cal77] A. P. Calderon, Cauchy integrals on Lipschitz curves and related operators, Proc. Nat. Acad. Sci. U.S.A. 74 (1977), 1324-1327. MR 57:6445

[CD93] M. Costabel and M. Dauge, Construction of corner singularities for Agmon-DouglisNirenberg elliptic systems, Math. Nachr. 162 (1993), 209-237. MR 94k:35090

[CMM82] R. R. Coifman, A. McIntosh, and Y. Meyer, L'integrale de Cauchy definit un operateur borne sur $L^{2}$ pour les courbes Lipschitziennes, Ann. of Math. 116 (1982), 361-387. MR 84m: 42027

[Dah80] B. E. J. Dahlberg, Weighted norm inequalities for the Lusin area integral and the nontangential maximal function for harmonic functions in Lipschitz domains, Studia Math 65 (1980), 297-314. MR 82f:31003

$\left[\mathrm{DK}^{+}\right] \quad$ B. E. J. Dahlberg, C. E. Kenig, J. Pipher, and G. C. Verchota, Area integral estimates and maximum principles for higher order elliptic equations and systems, Ann. Inst. Fourier (Grenoble), 47 (1997), no. 5, 1425-1461. MR 98m:35045

[FJR78] E. B. Fabes, M. Jodeit, and N. M. Riviere, Potential techniques for boundary value problems on $C^{1}$ domains, Acta Math. 141 (1978), 165-186. MR 80b:31006

[FS72] C Fefferman and E. Stein, $H^{p}$ spaces of several variables, Acta Math 129 (1972), 137-193. MR 56:6263

[Jou83] Jean-Lin Journé, Calderón-Zygmund Operators, Pseudo-Differential Operators and the Cauchy Integral of calderón, Lecture Notes in Mathematics, vol. 994, SpringerVerlag, Berlin Heidelberg New York Tokyo, 1983. MR 85i:42021

[Ken86] C. E. Kenig, Elliptic boundary value problems on Lipschitz domains, Beijing lectures in harmonic analysis, Annals of Math. Studies 112 (1986), 131-183. MR 88a:35066

[KKPT98] H. Koch, C. Kenig, J. Pipher, and T. Toro, A new approach to estimates for harmonic measure, and applications to nonsymmetric elliptic equations, preprint.

[Koz90] V. A. Kozlov, On the singularities of solutions of the Dirichlet problem for elliptic equations in the neighborhood of corner points, Leningrad Math. J. 1 (1990), 967-982.

[KWCQ85] Hua Loo Keng, Lin Wei, and Wu Ci-Quian, Second-order systems of partial differential equations in the plane, Pitman Advanced Publishing Program, Boston London Melbourne, 1985. MR 88m:35002

[PV95] J. Pipher and G. C. Verchota, Dilation invariant estimates and the boundary Garding inequality for higher order elliptic operators, Ann. Of Math. 142 (1995), 1-38. MR 96g:35052

[Ver84] G. C. Verchota, Layer potentials and regularity for the Dirichlet problem for Laplace's equation in Lipschitz domains, J. Funct. Anal. 59 (1984), no. 3, 572-611. MR 86e: 35038

[VV97] G. C. Verchota and Andrew L. Vogel, Nonsymmetric systems on nonsmooth planar domains, Trans. Amer. Math. Soc. 349 (1997), no. 11, 4501-4535. MR 98c:35040

Department of Mathematics, Syracuse University, Syracuse, New York 13244

E-mail address: alvogel@syr.edu 